\title{
ON THE LU QI-KENG CONJECTURE
}

\author{
NOBUYUKI SUITA AND AKIRA YAMADA
}

ABSTRACT. We shall give a complete answer to the Lu Qi-keng conjecture for finite Riemann surfaces. Our result is that every finite Riemann surface which is not simply-connected is never a Lu Qi-keng domain, i.e. the Bergman kernel $K(z, t)$ of it has zeros for suitable $t$ 's.

1. Let $\Omega$ be a Riemann surface and let $\Gamma$ be the Hilbert space of analytic differentials with finite norm on $\Omega$. Unless $\Omega$ is conformally equivalent to the Riemann sphere less a compact set of capacity zero, $\Gamma$ contains nontrivial differentials and the Bergman kernel of $\Omega$, denoted by $K(z, \bar{t}) d z d \bar{t}$ is not identically equal to zero. It is characterized by the reproducing property

$$
\alpha(z)=\frac{1}{2 i} \iint_{\Omega} K(z, \bar{t}) d \bar{t} \wedge \alpha(t) d t, \quad \alpha(t) d t \in \Gamma .
$$

Suppose that $\Omega$ has the Green's function $g(z, t)$. Then $K(z, \vec{t})$ is given by

$$
K(z, \bar{t})=-\frac{2}{\pi} \frac{\partial^{2} g(z, t)}{\partial z \partial \bar{t}} .
$$

This identity is due to Schiffer [3].

The following conjecture is called the Lu Qi-keng conjecture for a domain $\Omega \subset C^{n}$ by Skwarczynski [4]: The Bergman kernel $K(z, \bar{t})$ of $\Omega$ has no zeros in $\Omega \times \Omega$. This conjecture relates to a regularity problem of the invariant metric [1], [4]. He proved that some annulus $\left(\subset C^{1}\right)$ is not a Lu Qi-keng domain [4] and later Rosenthal showed that nor is every annulus [2].

2. We prove

THEOREM. Let $\Omega$ be a finite open Riemann surface which is not simplyconnected. Then the Bergman kernel $K(z, \bar{t})$ of $\Omega$ has exactly $n+2 p-1$ zeros for suitable $t$ 's. Here $n$ and $p$ denote the numbers of the boundary contours and the genus of $\Omega$, respectively.

Proof. Let $L(z, t) d z d t$ be the adjoint kernel of $K(z, \bar{t}) d z d \bar{t}$ given by

$$
L(z, t)=-\frac{2}{\pi} \frac{\partial^{2} g(z, t)}{\partial z \partial t}
$$

Received by the editors January 5, 1976.

AMS (MOS) subject classifications (1970). Primary 30A31.

Key words and phrases. Kernel function, Bergman kernel, Riemann surface.

(1) American Mathematical Society 1976 
which is a meromorphic differential on $\Omega$ with respect to $z$ and $t$ [3]. We denote by $\hat{\Omega}$ the double of $\Omega$. Then the differentials $K(z, \bar{t}) d z$ and $L(z, t) d z$ with fixed $t \in \Omega$ are meromorphically extended to $\hat{\Omega}$ by the relations:

$$
\begin{aligned}
& \alpha(z) d z= \begin{cases}\frac{K(z, \bar{t}) d z}{-L\left(z^{*}, t\right) d z} & \text { for } z \in \bar{\Omega},\end{cases} \\
& \beta(z) d z=\left\{\begin{array}{ll}
\frac{L(z, t) d z}{-K\left(t, \bar{z}^{*}\right) d z} & \text { for } z \in \bar{\Omega},
\end{array},\right.
\end{aligned}
$$

Note that $z$ and $t$ are used as local parameters and $z^{*}$ denotes the image of $z$ under the canonical involutive map of $\hat{\Omega}$.

It is well known that the quadratic differential $\alpha \beta d z^{2}$ is a positive differential on $\Omega$. Since $L(z, t) d z$ has one double pole at $t, \alpha \beta d z^{2}$ has $4 p+2 n-2$ zeros on $\bar{\Omega}$, multiplicities being counted by half on $\partial \Omega$. It is easily verified that the $t$ can be chosen at a point on $\partial \Omega$ and that the properties mentioned above still hold. We take a local parameter about $t \in \partial \Omega$ on a half neighborhood so that the boundary on $\partial \Omega$ and $t$ correspond to a segment on the real axis and to the origin respectively. Then for $t=x+i y$,

$$
\begin{aligned}
& \left.\frac{\partial g(z, t)}{\partial \bar{t}}\right|_{t=0}=\left.\frac{i}{2} \frac{\partial g(z, t)}{\partial y}\right|_{t=0}, \\
& \left.\frac{\partial g(z, t)}{\partial t}\right|_{t=0}=-\left.\frac{i}{2} \frac{\partial g(z, t)}{\partial y}\right|_{t=0} .
\end{aligned}
$$

Hence we have

$$
K(z, \bar{t}) d z=-L(z, t) d z, \quad z \in \Omega, t \in \partial \Omega .
$$

On the other hand,

$$
H(z)=\partial g(z, t) /\left.\partial y\right|_{t=0}
$$

is a positive harmonic function on $\Omega$ and vanishes identically on $\partial \Omega$ except for $z=t$. Since $H(z)$ can be harmonically extended to $\hat{\Omega}$ except for $z=t$, the meromorphic differential $d H$ has $2 p+n-1$ zeros in $\Omega$. Note that $H(z)$ has no critical points on $\partial \Omega$ and that $d H$ has one double pole at $t$. This means that $K(z, \bar{t})$ and $L(z, t)$ both have $2 p+n-1$ zeros for $t \in \partial \Omega$.

We let the reference point $\tau$ of $K(z, \bar{\tau}) d z$ tend to $t \in \partial \Omega$. Then $\{K(z, \bar{\tau})\}$ tends to $K(z, \bar{t})$ uniformly on every compact set in $\Omega$. Thus by Hurwitz's theorem the zeros of $K(z, \bar{\tau})$ cluster on the zeros of $K(z, \bar{t})$. This completes the proof.

3. If $\Omega$ is a compact Riemann surface, the Bergman kernel $K(z, \bar{t}) d z$ is an analytic differential on $\Omega$. In this case, $K(z, \bar{t})$ has exactly $2(p-1)$ zeros if the genus $p$ of $\Omega$ is greater than 1 . 


\section{REFERENCES}

1. Lu Qi-keng, On Kaehler manifolds with constant curvature, Acta Math. Sinica 16 (1966), 269-281 = Chinese Math.-Acta 8 (1966), 283-298. MR 34 \#6806.

2. Paul Rosenthal, On the zeros of the Bergman function in doubly-connected domains, Proc. Amer. Math. Soc. 21 (1969), 33-35. MR 39 \#25.

3. M. Schiffer, The kernel function of an orthonormal system, Duke Math. J. 13 (1946), 529-540. MR 8, 371.

4. M. Skwarczynski, The invariant distance in the theory of pseudoconformal transformations and the Lu Qi-keng conjecture, Proc. Amer. Math. Soc. 22 (1969), 305-310. MR 39 \# 5826.

Department of Mathematics, Tokyo Institute of Technology, Oh-Okayama, Meguro$\mathrm{KU}$, TOKYO, JAPAN 